\title{
Bradycardia as the First Sign of Brain Tumor: A Case Report
}

\author{
Ammar Kamil ${ }^{\mathrm{a}, \mathrm{c}}$, Hazim Ghazzay ${ }^{\mathrm{b}}$
}

\begin{abstract}
Sinus bradycardia can be normal in specific instances. However, it had been sporadically documented in the literature as the first sign of a brain tumor. We report a case of a young man who was complaining of dizziness without any neurological sign, and found to have a sinus bradycardia. No underlying cardiac cause was found and he had non-significant medication history. Surprisingly his head computed tomography showed a fronto-temporal brain tumor. This highlights that bradycardia can be the first and the only warning sign for brain tumors, which is likely to be missed.
\end{abstract}

Keywords: Brain tumor; Sinus bradycardia; Increased intracranial pressure

\section{Introduction}

A growing tumor in the brain is commonly associated with edema and increased intracranial pressure (ICP). Common signs and symptoms due to increased ICP or brain edema include headache, nausea, vomiting, focal neurological deficit and an altered mental function [1,2]. Sinus bradycardia (sinus rhythm of less than $60 \mathrm{bpm}$ ), which can be a normal phenomenon, has been only sporadically documented in the literature as the first signs of a brain tumor [3, 4].

We present the case of a young man who was complaining of dizziness not responding to over the counter medications. On examination, the patient was normotensive with no postural hypotension. There were no abnormal cardiological or neurological signs, and the fundoscopy was inconclusive. The resting electrocardiogram was unremarkable except for a sinus bradycardia of (40 beats/min), accelerating normally with exercise. A computed tomography (CT) of the head surprisingly showed a brain tumor.

This highlights that bradycardia can be the first and the only warning sign of brain tumors, which is more likely to be

Manuscript submitted March 6, 2018, accepted March 20, 2018

aUniversity of Nebraska Medical Center, Omaha, NE, USA

${ }^{b}$ Department of Internal Medicine, Al-Ramadi Teaching Hospital, Anbar, Iraq ${ }^{\mathrm{c} C}$ Corresponding Author: Ammar Kamil, University of Nebraska Medical Center, Omaha, NE 68198-7400, USA. Email: ammar.kamil@unmc.edu

doi: https://doi.org/10.14740/jmc3032w missed.

\section{Case Report}

A 41-year-old man, without a relevant medical history, not taking any medication, attended the outpatient clinic complaining of dizziness for 3 weeks, not associated with nausea, vomiting, blackout, or any other neurological symptoms. No chest pain, no dyspnea and no improvement had been noticed, although he consulted several physicians and tried several over the counter medications.

On examination, the patient had normal mental status, oriented to persons, places and time, his resting blood pressure was $137 / 83 \mathrm{~mm} \mathrm{Hg}$, resting respiratory rate was 17 respirations/min, and no signs of increase ICP was noticed by the fundoscopy. Cranial nerves examination was normal and no limbs weakness was found. The only finding was the sinus bradycardia of 40 beats/min as shown in Figure 1. We admitted him to the coronary care unit for close monitoring. Further workup was performed, including echocardiogram which was normal and stress test which showed a normal acceleration of the heart rate without any signs or symptoms of ischemia. Thereafter, we decided to perform a CT of the head, to exclude any intracranial cause, which surprisingly showed a brain tumor of about $5 \times 4 \mathrm{~cm}$ in size at the right fronto-temporal region as shown in Figure 2.

Dexamethasone was started and the patient referred to the neurosurgery department for further surgical management.

\section{Discussion}

Sinus bradycardia (sinus rhythm of less than $60 \mathrm{bpm}$ ) can be normal in specific instances. However, it could be due to cardiac causes such as ischemic heart disease, sick sinus syndrome, or non-cardiac causes such as metabolic causes, medications, and brain tumors [5-7].

Many investigations led to understand the neurophysiology of the cardiovascular control and showed the importance of the several regions of the nervous system (specially the brainstem, thalamus, hypothalamus and amygdala), in the pathophysiology of serious cardiac dysrhythmias $[8,9]$. However, bradycardia in specific has been reported in a patient with a temporal lobe tumor by Van Der Sluijs in 2004 [1]. Three years later, Ki-Hoon Park and his coworkers [4] related the origin of bradycardia in their patient to a brain tumor in the frontal lobe depending on several studies. These studies suggested a fron- 


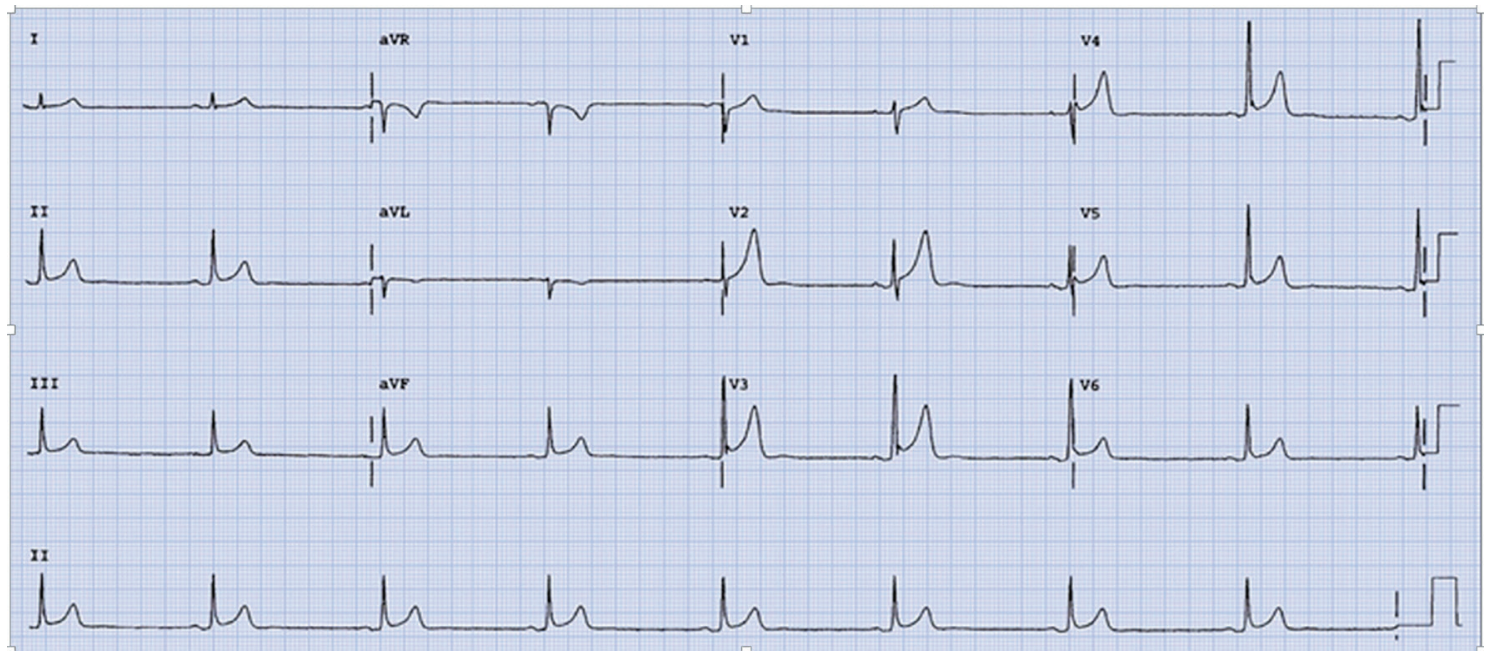

Figure 1. EKG of a 41-year-old man shows sinus bradycardia without any signs of ischemia.

tal lobe influence on the autonomic cardiovascular regulation [10-13], and the electrical stimulation of that lobe produces changes in the heart rate [14-16]. Accordingly, it is believed that the origin of the bradycardia in our patient was the frontotemporal lobe tumor, which is shown by the head CT.

On the other hand, the elevation of ICP with the subsequent decrease of cerebral perfusion pressure significantly induced a vigorous cerebro-protective neuroendocrine system activation that can lead to the neurogenic stunned myocardium [17], which is defined as myocardial injury and dysfunction occurring after diverse types of acute brain injury as a result of imbalance of the autonomic nervous system. The spectrum of observed cardiac abnormalities includes electrocardiographic changes, myocardial necrosis, both systolic and diastolic dysfunction and arrhythmia including bradycardia. Although management should include careful cardiac monitoring, treatments should generally focus on the underlying neurologic process to maximize neurologic recovery [18].

\section{Conclusion}

This case report reveals a neglected cause of sinus bradycardia, suggesting that awareness is needed for suspecting increased ICP even if there were no other signs.

\section{References}

1. van der Sluijs BM, Renier WO, Kappelle AC. Brain tumour as a rare cause of cardiac syncope. J Neurooncol. 2004;67(1-2):241-244.
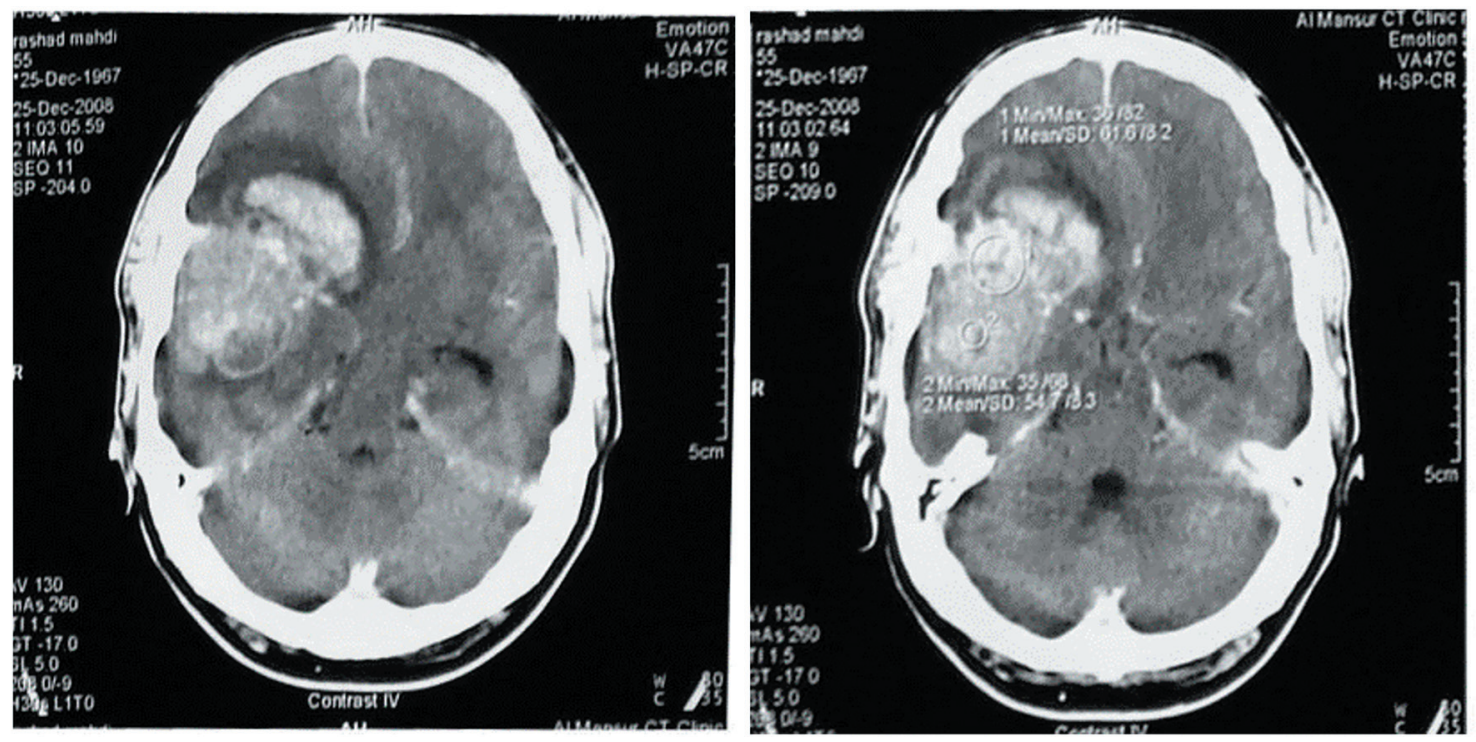

Figure 2. A cross-sectional contrast CT scan of the head showing a hyperdense lesion at the right fronto-temporal region about $5 \times 4 \mathrm{~cm}$ in size, with surrounding brain edema and causing a midline shift of brain structure. A diagnosis of glioma had made. 
2. Bedi H, Mitera G, Sahgal A, Pirouzmand F, Bilbao J, Sinclair E, Fitch A, et al. Neurosurgical rescue of bradycardia induced by intracerebral hypertension: a case report and review of the literature. J Palliat Med. 2009;12(6):563565.

3. Spodick DH. Normal sinus heart rate: sinus tachycardia and sinus bradycardia redefined. Am Heart J. 1992;124(4):1119-1121.

4. Ki-Hoon Park, Sung-Ho Her, Jong-Min Lee, Hee-Jeoung Yoon, Jung-Yeon Chin, Jun-Han Jeon, Ye-Lee Park, et al. Brain tumor is a rare cause of both bradycardia and seizure. Korean Circulation J. 2007;37:449-452.

5. Semelka M, Gera J, Usman S. Sick sinus syndrome: a review. Am Fam Physician. 2013;87(10):691-696.

6. Wung SF. Bradyarrhythmias: clinical presentation, diagnosis, and management. Crit Care Nurs Clin North Am. 2016;28(3):297-308.

7. Guasch E, Mont L. Diagnosis, pathophysiology, and management of exercise-induced arrhythmias. Nat Rev Cardiol. 2017;14(2):88-101.

8. Oppenheimer S. The anatomy and physiology of cortical mechanisms of cardiac control. Stroke. 1993;24(12 Suppl):I3-5.

9. Oppenheimer S, Norris JW. Cardiac manifestations of acute neurological lesions. In: Aminoff MJ (ed) Neurology and General Medicine. Churchill Livingstone, Philadelphia. 2001; p. 171-185.

10. Mascia A, Quarato PP, Sparano A, Esposito V, Sebastiano
F, Occhiogrosso G, Di Gennaro G. Cardiac asystole during right frontal lobe seizures: a case report. Neurol Sci. 2005;26(5):340-343.

11. Tinuper P, Bisulli F, Cerullo A, Carcangiu R, Marini C, Pierangeli G, Cortelli P. Ictal bradycardia in partial epileptic seizures: Autonomic investigation in three cases and literature review. Brain. 2001;124(Pt 12):2361-2371.

12. Leung H, Schindler K, Kwan P, Elger C. Asystole induced by electrical stimulation of the left cingulate gyrus. Epileptic Disord. 2007;9(1):77-81.

13. Sebastiani L, Ghelarducci B. Medial prefrontal cortex early lesion effects on classical conditioned bradycardia. Exp Brain Res. 2002;147(2):264-267.

14. Oppenheimer SM, Cechetto DF, Hachinski VC. Cerebrogenic cardiac arrhythmias. Cerebral electrocardiographic influences and their role in sudden death. Arch Neurol. 1990;47(5):513-519.

15. Pool JL, Ransohoff J. Autonomic effects on stimulating rostral portion of cingulate gyri in man. J Neurophysiol. 1949;12(6):385-392.

16. Wall PD, Davis GD. Three cerebral cortical systems affecting autonomic function. J Neurophysiol. 1951;14(6):507517.

17. Agrawal A, Timothy J, Cincu R, Agarwal T, Waghmare LB. Bradycardia in neurosurgery. Clin Neurol Neurosurg. 2008;110(4):321-327.

18. Nguyen H, Zaroff JG. Neurogenic stunned myocardium. Curr Neurol Neurosci Rep. 2009;9(6):486-491. 